\title{
CrystEngComm
}

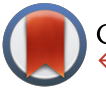

CrossMark $\leftarrow$ click for updates

Cite this: CrystEngComm, 2016, 18, 3015

Received 29th March 2016, Accepted 4th April 2016

DOI: $10.1039 / c 6 c e 00698 a$

\section{Solid-state isolation of a unique, small-molecule, supra-heterodimer of large hexameric assemblies of C-methylcalix[4]resorcinarene $\uparrow$}

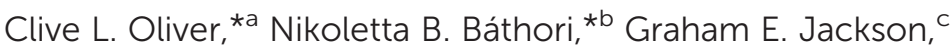 \\ David Kuter ${ }^{\mathrm{C}}$ and Dyanne L. Cruickshank ${ }^{\mathrm{a}}$
}

www.rsc.org/crystengcomm

The well-known host molecule $C$-methylcalix[4]resorcinarene is shown to exist in two unique hexameric arrangements within the same crystal. These two hexameric assemblies are linked via a water molecule into a supra-heterodimer.

Nature is able to produce large, supramolecular assemblies of macromolecules with intricate complexities such as found in viruses, cellular membranes and ribosomes. ${ }^{1-3}$ These complicated structures are held together by non-covalent interactions which are ultimately crucial in the functioning of these complex biological systems. Small-molecule supramolecular chemists are inspired by these complex supramolecular systems in nature, however, large, synthetic, multicomponent $(n>3)$ supramolecular assemblies which enclose chemical space are relatively rare phenomena in the field of small-molecule, supramolecular chemistry. Atwood and MacGillivray reported the first example of such an assembly by showing that the bowl-shaped host molecule $C$-methylcalix[4]resorcinarene 1a (Scheme 1) can spontaneously assemble in a nitrobenzene solution to form a large, chiral, supramolecular assembly consisting of 6 molecules of 1a and 8 water molecules, the latter 'stitching' molecules of 1 a into a hexameric assembly, $1 \mathrm{a}_{6} \cdot\left(\mathrm{H}_{2} \mathrm{O}\right)_{8}$ via $\mathrm{O}-\mathrm{H}^{\cdots} \mathrm{O}$ hydrogen bonds. ${ }^{4}$ Despite the approximately 125 structures reported since this discovery containing 1a co-crystallised with various guest and/or solvent molecules, ${ }^{5}$ only one similar hexameric assembly of 1a was reported by Holman et al. where 6 of the 8 water molecules were replaced by 2-ethylhexanol (2-EH) molecules. ${ }^{6}$ However, large hexameric

${ }^{a}$ Centre for Supramolecular Chemistry, Department of Chemistry, University of Cape Town, Rondebosch, 7701, South Africa. E-mail: clive.oliver@uct.ac.za

${ }^{b}$ Crystal Engineering Unit, Department of Chemistry, Faculty of Applied Sciences, Cape Town, 8000, South Africa. E-mail: bathorin@cput.ac.za;

Fax: +27 21460 3854; Tel: +27214608354

${ }^{c}$ Department of Chemistry, University of Cape Town, Rondebosch, 7701, South Africa

$\dagger$ Electronic supplementary information (ESI) available: Experimental detail, X-ray data, TGA analyses, NOE NMR detail. CCDC 1430586. For ESI and crystallographic data in CIF or other electronic format see DOI: 10.1039/c6ce00698a supramolecular assemblies have been reported for the structurally related pyrogallolarenes (such as $2 \mathbf{a}$ in Fig. 1), which have three hydroxyl groups per aromatic ring, the additional hydroxyl group negating the need for a stitching solvent molecule. $^{7-12}$

Recently, much research has been focussed on linking the pyrogallolarene molecules of the hexameric assemblies with metal ions by deprotonation of the hydroxyl groups of the pyrogallolarene molecules, resulting in metallocapsule analogues of the 'purely' supramolecular assemblies. ${ }^{13-16}$ Structurally related calix[4]arenes have also been shown to form large supramolecular assemblies that enclose chemical space, however these assemblies are dodecameric with the host molecule orientation being reversed when compared to the hexameric resorcinarene and pyrogallolarene assemblies. Here the truncated cone shape of the molecules, together with their "up-up" arrangement was suggested to be the determining factor in the formation of large spherical

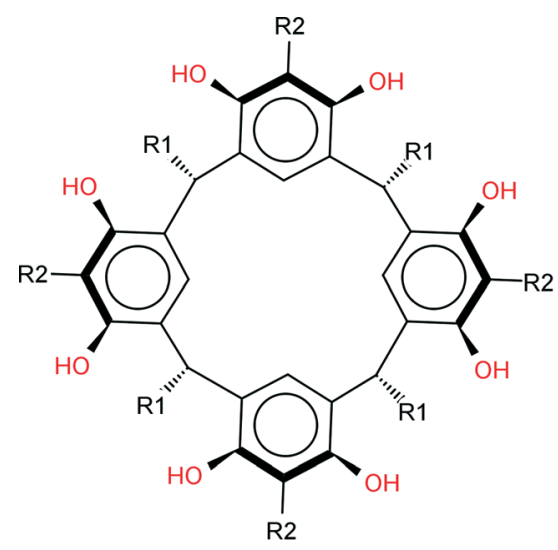
1a $\quad \mathrm{R} 1=\mathrm{CH}_{3}$
$\mathrm{R} 2=\mathrm{H}$
1b $\mathrm{R} 1=\left(\mathrm{CH}_{2}\right)_{10} \mathrm{CH}_{3}$
$\mathrm{R} 2=\mathrm{H}$
2a $\mathrm{R} 1=\mathrm{CH}_{2} \mathrm{CH}\left(\mathrm{CH}_{3}\right)_{2}$
$\mathrm{R} 2=\mathrm{OH}$

Scheme 1 Chemical structures. 

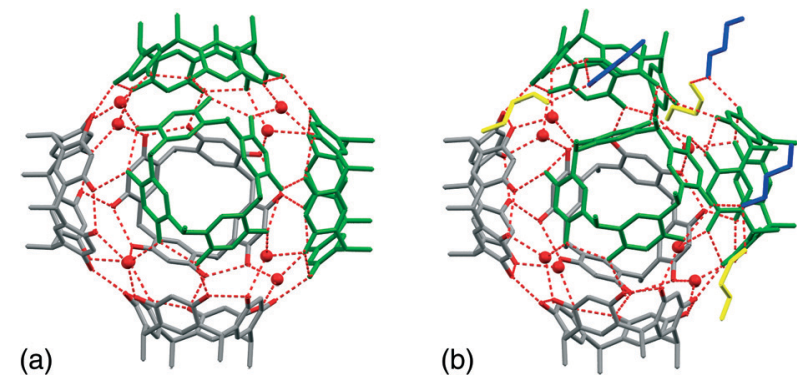

Fig. 1 Hexameric assemblies of 1 a showing the (a) regular hexameric assembly $\mathbf{A}$ and the (b) more irregular assembly $\mathbf{B}$ with its associated 1-BuOH molecules.

assemblies, rather than on the basis of hydrogen bonding considerations. ${ }^{17}$ The geometries of these dodecameric assemblies have been compared to that of icosahedral viruses or 'reverse micelles'. ${ }^{18,19}$ It is notable that in all these assemblies only one type of assembly per crystal structure was isolated in the solid-state. Here we present a crystallisation of 1a from 1-butanol 1-BuOH, which yielded two different types of hexameric assemblies within the same crystal structure. Furthermore, the two unique assemblies are linked into a heterodimer of hexameric assemblies which we entitle a supra-heterodimer. To the best of our knowledge, the isolation of two different large, supramolecular assemblies $(n>3)$ within the same crystal structure has not been observed before and neither has identical large supramolecular assemblies been shown to link into discrete units. ${ }^{5}$

Structure solution and refinement revealed that the asymmetric unit consists of four $1 \mathrm{a}$, four $\mathbf{1 - B u O H}$ and nine water molecules. Two independent hexameric assemblies are formed which will be referred to as assembly $\mathbf{A}$ and $\mathbf{B}$ hereinafter (Fig. 1). Both assemblies consist of six molecules of 1a with a water molecule 'stitching' three adjacent 1a molecules into a trigonal arrangement via water $\cdots 1$ a $\mathrm{O}-\mathrm{H} \cdots \mathrm{O}$ hydrogen bonding, as in the case of the Atwood and Holman hexamers. These water molecules can be considered as residing on the vertices of a cube with the 1a molecules residing on the faces of such a cube. Assembly A has eight molecules on all eight vertices of the cube whilst assembly $\mathbf{B}$ has seven, the eighth water molecule being absent due to the closer arrangement of three 1a molecules at this position (intersection of 'green' molecules in Fig. 1b). Assembly $\mathbf{B}$ seems to be in a partially 'collapsed' state with three 1a molecules being tilted by $\sim 13^{\circ}$ relative to the corresponding molecules in assembly $\mathbf{A}$ ('green' molecules in Fig. 1a and b). Two unique 1-BuOH molecules are associated with assembly $\mathbf{B}$ at three locations around the assembly. These 1-BuOH molecules are hydrogen bonded to each other, with one in turn being hydrogen bonded to a molecule of 1a, whilst a water molecule is located further into the cavity at this position. The presence of the butanol molecules seem to disrupt the regular arrangement of $\mathbf{1 a}$ in assembly $\mathbf{B}$, since the water molecules close to the 1-BuOH molecules are not replaced (as in the case of the Holman hexamer) but instead located deeper into the interior of the hexameric assembly.
This disruption may also be responsible for the exclusion of the water molecule at the eighth vertex of the cube as three 1a molecules are involved in direct $1 \mathrm{a} \cdots 1 \mathrm{a} \mathrm{O}-\mathrm{H} \cdots \mathrm{O}$ hydrogen bonding at this point. Thus, the partially 'collapsed' state of assembly B could be a 'snapshot' of the movement of butanol molecules from the interior to the exterior of these assemblies. The program MSRoll $^{20}$ calculated that assembly A (using a probe radius of $1.5 \AA$ ) has a significantly larger interior volume of $1306 \AA^{3}$ when compared to the interior volume of $1186 \AA^{3}$ of assembly B (Fig. S1, ESI $\dagger$ ) which illustrates the deformation of assembly B. Holman et al. compared their assembly to that of Atwood et al. from a hydrogen-bonding perspective as well as the arrangement of 1a molecules, considering it as a 14-component assembly with formula $\mathbf{1 a}_{6} \cdot(\mathbf{2 E H})_{6}$ $\cdot\left(\mathrm{H}_{2} \mathrm{O}\right)_{2}$ with slightly fewer hydrogen bonds than the $\mathbf{1 a}_{6}$ $\cdot\left(\mathrm{H}_{2} \mathrm{O}\right)_{8}$ assembly (58 vs. 60) of Atwood et al. Holman et al. also indicated that opposing molecules of $\mathbf{1 a}$ in their assembly were eclipsed whilst in the Atwood assembly they were rotated by $45^{\circ}$. In this respect assemblies A and B in our crystal structure resemble the Atwood assembly more closely in that the opposing resorcinarenes are rotated by $\sim 45^{\circ}$. Relative to the Atwood hexamer, the point group symmetries of assemblies A and B are relaxed from $O 432$ to $C 3$, which shows that assemblies A and B are also chiral, unlike the Holman assembly that is achiral with its $S_{6}$ point group symmetry. Assembly A is also a close match to the Atwood hexamer in terms of hydrogen bonding and similarly has 24 1a intramolecular, 12 1a $\cdots 1$ a intermolecular and 24 water $\cdots 1$ a $\mathrm{O}-\mathrm{H} \cdots \mathrm{O}$ hydrogen bonds. However, it has two additional water $\cdots$ water $\mathrm{O}-\mathrm{H} \cdots \mathrm{O}$ hydrogen bonds, one that links a 'vertex' water molecule to a terminal water molecule at one end of assembly $\mathbf{A}$ and one at the opposite end that links a 'vertex' water molecule to the water molecule that links the two assemblies (Fig. 2). This constitutes a total of 62 hydrogen bonds for assembly $\mathbf{A}$. Assembly B displays a more intricate hydrogen bonding pattern and has 24 1a intramolecular, 12 1a $\cdots 1$ a intermolecular and 21 water $\cdots 1$ a $\mathrm{O}-\mathrm{H} \cdots \mathrm{O}$ hydrogen bonds, the latter reduction in the number of hydrogen bonds being due to the exclusion of the eighth water molecule. In addition, it has 3 1-BuOH $\cdots 1$-BuOH, 3 1-BuOH $\cdots 1$ a and 1 water $\cdots$ water hydrogen bonds for a total of 64 hydrogen bonds. Together with the 3 hydrogen bonds that form between 3 1-BuOH molecules and the water molecule that links the two assemblies, a

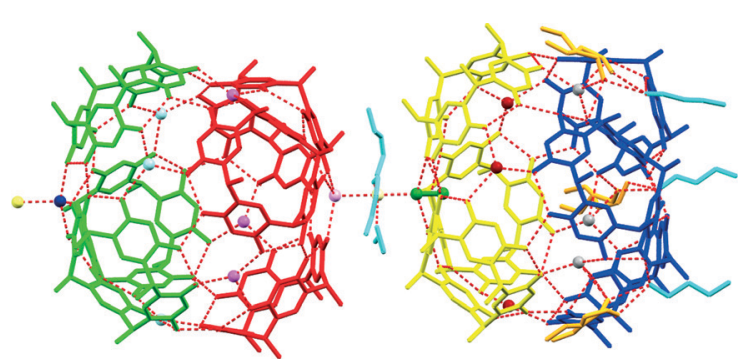

Fig. 2 The dimer of hexameric assemblies formed by two assemblies of A (left) and B (right) (symmetry-equivalent atoms are colour-coded). 
heterodimer of hexameric assemblies is formed; a 38component assembly with 129 hydrogen bonds and formula $\mathbf{1 a}_{12} \cdot\left(\mathrm{H}_{2} \mathrm{O}\right)_{17}(\mathbf{1}-\mathrm{BuOH})_{9}$, which we term a supra-heterodimer. Interestingly, although only one unique butanol molecule was modelled successfully in the cavity of a 1a molecule (with a site occupancy factor of 0.52 ) in assembly $\mathbf{A}$ (not shown in Fig. 1 or 2) the residual electron density maps did reveal that 1-BuOH molecules may be present in the cavities of the remaining three unique 1a molecules. The SQUEEZE routine in PLATON $^{21}$ calculated 269 electrons per asymmetric unit which would correspond to and additional 6.4 unmodelled 1-BuOH molecules. This is consistent with the diffuse electron density not being only confined to the interior of the assemblies as significant void space was indicated by Mercury $^{22}$ to exist between adjacent supra-heterodimers along the $c$-axis (Fig. 3). TGA analysis (Fig. S2, ESI $\dagger$ ) indicates a mass loss of $29.03 \%$ which is very close to the $29.33 \%$ solvent that the crystallographic model together with the proposed unmodelled 1-BuOH molecules suggests. Powder X-ray diffraction (Fig. S3, ESI $\dagger$ ) confirms that the single crystal structure is representative of the bulk material.

Solution NMR studies of $1 \mathrm{a}$ are challenging due to its sparing solubility in $\mathrm{CDCl}_{3}$, and therefore $\mathbf{1 b}$ is commonly used as an analogue. The ${ }^{1} \mathrm{H}$ NMR spectrum of $\mathbf{1 b}$ in the presence of 1-BuOH shows two distinct sets of resonances for 1-BuOH - the normal spectrum of the 'free' 1-BuOH and a series of peaks, shifted to high field, which are assigned to 'bound' 1-BuOH. Cohen et al. used the LED pulse sequence to measure the diffusion rate of 'bound' $1-\mathrm{BuOH}$ and found it to depend on the length of the eddy delay. ${ }^{23-25}$ This they ascribed to slow exchange between the two forms of 1-BuOH and therefore concluded that the $\mathbf{1 - B u O H}$ is not encapsulated inside the 1b cage, but rather, is bound to the cage. Instead of using diffusion rates, we have used selective NOE measurements. Irradiation of an NMR signal can result in cross relaxation, through space, to a neighbouring nucleus, via the nuclear Overhauser effect or via chemical exchange. Fig. 4 shows the results of a series of the selective NOE measurements. When the methyl of 'bound' $1-\mathbf{B u O H}$ is selectively irradiated (Fig. 4a) only the methyl of free 1-BuOH is affected. This must be the result of chemical exchange. When ${ }^{3} \mathrm{CH}_{2}$ of 'bound' 1-BuOH is irradiated (Fig. 4c), again the free ${ }^{3} \mathrm{CH}_{2}$ of

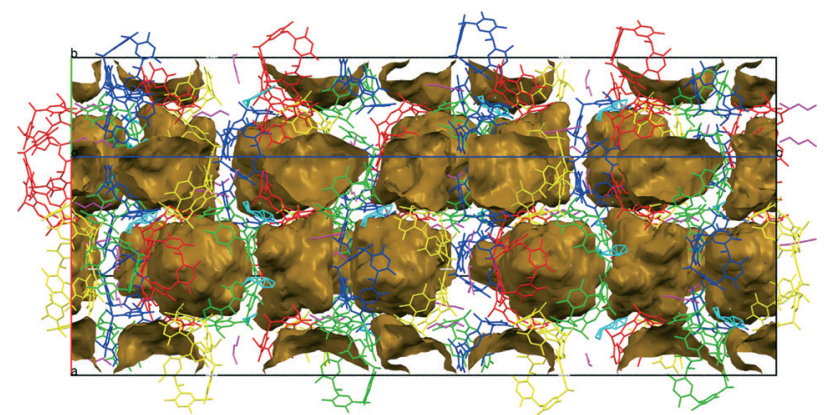

Fig. 3 Packing diagram showing hexamer interior spaces as well cavities formed between dimers.

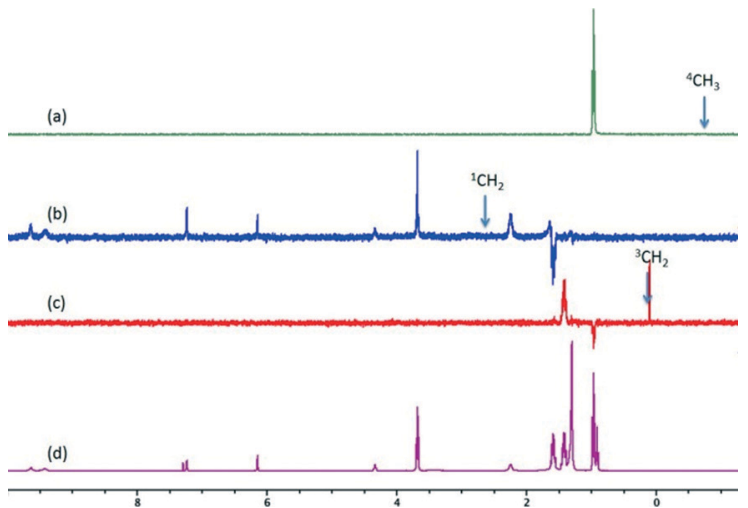

Fig. 4 The NOE spectra where protons of 'bound' 1-BuOH were selectively irradiated for (a) ${ }^{4} \mathrm{CH}_{3}$ (b) ${ }^{1} \mathrm{CH}_{2}$ and (c) ${ }^{3} \mathrm{CH}_{2}$. (d) The normal spectrum of $1-\mathrm{BuOH}$ and $1 \mathrm{~b}$.

1-BuOH is effected through chemical exchange. However, irradiation of bound ${ }^{1} \mathrm{CH}_{2}$ (Fig. 4b) leads to enhancement of not only free $\mathbf{1 - B u O H}$ but also several peaks from $\mathbf{1 b}$. The enhancement of the $\mathbf{1 b}$ resonances must be a through space, NOE effect, providing direct evidence for the association of 1-BuOH with the 1 b. Furthermore, since only ${ }^{1} \mathrm{CH}_{2}$ shows an NOE effect the 1-BuOH must be oriented with the alkyl chain pointing away from the hexameric assembly. This is the same orientation as we find in the crystal structure of 1a. It should be pointed out, however, that using chemical shift arguments, Cohen et al. concluded that the tail pointed towards the interior of the assembly. ${ }^{23}$ In our opinion, it is probably unlikely that a similar supramolecular heterodimer observed in the solid-state for $\mathbf{1 a}$ is present for $\mathbf{1 b}$ in solution due to the long undecyl chain of $\mathbf{1 b}$ which would prevent the close approach of two hexameric assemblies.

In summary, two unique hexameric assemblies of 1a have been isolated in the same crystal structure. These assemblies are linked via a water molecule into a heterodimeric, supramolecular assembly consisting of 38 components held together by 129 hydrogen bonds. This represents an advance in the hierarchical structure of these types of small-molecule assemblies. Although these systems are not close to that of their biological counterparts in terms of size and complexity, they do represent important steps in the synthetic, small-molecule supramolecular chemist's attempts to mimick nature's supramolecular organization. Future studies will concentrate on assessing the role of water or other solvent molecules in the co-crystallisation of different types of large, supramolecular assemblies within the same crystal structure, and whether a general synthetic method can be developed for the simultaneous isolation of different large supramolecular assemblies.

\section{Acknowledgements}

We are grateful to the University of Cape Town and the South African National Research Foundation for funding support. We also thank Professor Ton Spek and Professor George Sheldrick in helping out with .cif file issues which arose due to the long crystallographic $c$-axis. 


\section{Notes and references}

1 K. Ariga, J. P. Hill, M. V. Lee, A. Vinu, R. Charvet and S. Acharya, Sci. Technol. Adv. Mater., 2008, 9, 014109.

2 Z. Liu, J. Qiao, Z. Niu and Q. Wang, Chem. Soc. Rev., 2012, 41, 6178-6194.

3 D. A. Uhlenheuer, K. Petkau and L. Brunsveld, Chem. Soc. Rev., 2010, 39, 2817-2826.

4 L. R. MacGillivray and J. L. Atwood, Nature, 1997, 389, 469-472.

5 F. Allen, Acta Crystallogr., Sect. B: Struct. Sci., 2002, 58, 380-388.

6 O. Ugono and K. T. Holman, Chem. Commun., 2006, 2144-2146.

7 T. Gerkensmeier, W. Iwanek, C. Agena, R. Frohlich, S. Kotila, C. Nather and J. Mattay, Eur. J. Org. Chem., 1999, 2257-2262.

8 J. L. Atwood, L. J. Barbour and A. Jerga, Chem. Commun., 2001, 2376-2377.

9 L. Avram and Y. Cohen, J. Am. Chem. Soc., 2003, 125, 16180-16181.

10 G. W. V. Cave, J. Antesberger, L. J. Barbour, R. M. McKinlay and J. L. Atwood, Angew. Chem., Int. Ed., 2004, 43, 5263-5266.

11 Q. Zhang, R. D. Adams and D. Fenske, J. Inclusion Phenom. Macrocyclic Chem., 2005, 53, 275-279.

12 S. J. Dalgarno, N. P. Power, J. Antesberger, R. M. McKinlay and J. L. Atwood, Chem. Commun., 2006, 3803-3805.
13 N. P. Power, S. J. Dalgarno and J. L. Atwood, Angew. Chem., Int. Ed., 2007, 46, 8601-8604.

14 S. J. Dalgarno, N. P. Power and J. L. Atwood, Coord. Chem. Rev., 2008, 252, 825-841.

15 P. P. Cholewa and S. J. Dalgarno, CrystEngComm, 2014, 16, 3655-3666.

16 P. P. Cholewa and S. J. Dalgarno, Abstracts of Papers of the American Chemical Society, 2014, vol. 247.

17 G. W. Orr, L. J. Barbour and J. L. Atwood, Science, 1999, 285, 1049-1052.

18 J. L. Atwood, L. J. Barbour, S. J. Dalgarno, M. J. Hardie, C. L. Raston and H. R. Webb, J. Am. Chem. Soc., 2004, 126, 13170-13171.

19 K. Suwinska, B. Lesniewska, M. Wszelaka-Rylik, L. Straver, S. Jebors and A. W. Coleman, Chem. Commun., 2011, 47, 8766-8768.

20 M. L. Connolly, J. Mol. Graphics, 1993, 11, 139-141.

21 A. L. Spek, Acta Crystallogr., Sect. D: Biol. Crystallogr., 2009, 65, 148-155.

22 C. F. Macrae, I. J. Bruno, J. A. Chisholm, P. R. Edgington, P. McCabe, E. Pidcock, L. Rodriguez-Monge, R. Taylor, J. van de Streek and P. A. Wood, J. Appl. Crystallogr., 2008, 41, 466-470.

23 S. Slovak, L. Avram and Y. Cohen, Angew. Chem., Int. Ed., 2010, 49, 428-431.

24 S. Slovak and Y. Cohen, Chem. - Eur. J., 2012, 18, 8515-8520.

25 L. Avram and Y. Cohen, Chem. Soc. Rev., 2015, 44, 586-602. 